\title{
Conocimiento del derecho al servicio de interpretación de Lengua de Señas Argentina por pediatras que ejercen en la Ciudad Autónoma de Buenos Aires
}

\author{
Awareness of the right to Argentine Sign Language interpretation services \\ among pediatricians practicing in the Autonomous City of Buenos Aires
}

\author{
Sr. Lucas A. Walz $z^{a}$ Dr. Adrián M. Cutrib, Dr. Fernando A. Torres ${ }^{c}$, Prof. Claudio C. Riquelmed, \\ Prof. Verónica Gatto Bellorac y Dr. Fernando C. Ferrero ${ }^{c}$
}

\section{RESUMEN}

Introducción. Los obstáculos en la comunicación entre médicos y personas sordas/hipoacúsicas pueden comportarse como factores de riesgo para la salud. El servicio de un intérprete profesional de Lengua de Señas Argentina (IPLSA), según la Ley N. ${ }^{\circ} 26378$, podría mitigar esto.

Objetivo. Estimar la proporción de pediatras que conocen la Ley N..$^{\circ} 26378$ y explorar si se asocia a recomendar a un IPLSA. Población y métodos. Estudio transversal con cuestionario electrónico autoadministrado a pediatras registrados en la Sociedad Argentina de Pediatría, que ejercían en la Ciudad Autónoma de Buenos Aires.

Resultados. Se evaluaron 381 respuestas. El 15,1 \% conocía la Ley, y esto se asoció con recomendar a un IPLSA (OR: 3,7; IC $95 \%: 1,1-12,9 ; \mathrm{p}<0,05)$.

Conclusiones. La proporción de pediatras de la Ciudad Autónoma de Buenos Aires que conocía la ley fue el 15,1\%. Esto se asoció significativamente con recomendar a un IPLSA. Palabras clave: derecho a la salud, sordera, lenguaje de signos.

http: / / dx.doi.org/10.5546/ aap.2021.123

Texto completo en inglés:

http:/ / dx.doi.org/10.5546/ aap.2021.eng.123

Cómo citar: Walz LA, Cutri AM, Torres FA, Riquelme CC, et al. Conocimiento del derecho al servicio de interpretación de Lengua de Señas Argentina por pediatras que ejercen en la Ciudad Autónoma de Buenos Aires. Arch Argent Pediatr 2021;119(2):123-128.

a. Universidad de Yale.

b. Escuela de Educación Especial "Julia Ofelia Cubilla" No 7, Resistencia, Chaco.

c. Hospital General de Niños "Pedro de Elizalde", Ciudad Autónoma de Buenos Aires, Argentina.

d. Unidad Educativa de Gestión Privada Nº 172 "Deolindo Felipe Bittel", Resistencia, Chaco, Argentina.

Correspondencia:

Dr. Adrián M. Cutri: adriancutri@gmail.com

Financiamiento: Trabajo realizado por un sistema de becas de la Universidad de Yale, Gary Stein Summer Fellowship,

Yale College Fellowships for Research in Global Health Studies,

Steven Clark Senior Essay Travel Grant.

Conflicto de intereses: Ninguno que declarar.

Recibido: 28-5-2020

Aceptado: 6-10-2020

\section{INTRODUCCIÓN}

La pérdida de la audición se encuentra entre las discapacidades de mayor prevalencia, ${ }^{1}$ pues afecta a más de 466 millones de personas en el mundo. ${ }^{2}$ El Ministerio de Salud de la República Argentina estima que la discapacidad auditiva representa el $18 \%$ de las discapacidades a nivel nacional. ${ }^{3}$ En nuestro país, muchas personas sordas o hipoacúsicas (PSH) utilizan la Lengua de Señas Argentina (LSA) como método de comunicación.

Los obstáculos en la comunicación son un factor de riesgo para la salud entre las PSH. Investigaciones en el Reino Unido demostraron mayores probabilidades de tener asma, hipertensión, obesidad y depresión en estas personas. ${ }^{4}$ Además, tienen mayores probabilidades de percibir que sus necesidades de atención médica no están cubiertas. ${ }^{5} \mathrm{Si}$ bien pueden existir razones biológicas subyacentes, la desigualdad en el acceso a los servicios de salud podría explicar esta percepción. ${ }^{6}$

Las PSH tienen mayores probabilidades de tener dificultades para comunicarse con sus médicos, entenderlos y sentirse cómodas con ellos. Además, la comunicación ineficaz entre médicos y PSH limita la atención en salud. ${ }^{7}$ El servicio de un intérprete profesional que pueda comunicarse a través de la lengua de señas podría mitigar esas limitaciones.

La Argentina aprobó la Convención de los Derechos de las Personas con Discapacidad (Ley $\mathrm{N}^{\circ} 26378$ ) y, posteriormente, se le dio jerarquía constitucional (Ley $\mathrm{N}^{\circ} 27044$ ), con lo que se reconocieron los derechos de las personas con discapacidad. ${ }^{8}$ Esta norma estipula proporcionar a un intérprete profesional de LSA (IPLSA) gratuito para facilitar el acceso de PSH a instalaciones públicas. Además, la Convención de los Derechos del Niño (Ley No 23849) enfatiza las obligaciones del Estado para prestar asistencia apropiada a los padres para el desempeño de sus 
responsabilidades de crianza. ${ }^{9}$ La ausencia de un IPLSA en la consulta implica la conculcación de derechos de PSH y de sus hijos.

Un cuestionario realizado a padres sordos/ hipoacúsicos en la Ciudad Autónoma de Buenos Aires (CABA) y en Chaco reveló que el 15,4 \% había tenido alguna vez un IPLSA durante la consulta y ninguno había conseguido este servicio por parte del Estado. ${ }^{10}$ Explorar si los médicos conocen la Ley $N^{\circ} 26378$ y si brindan información sobre el derecho de contar con un IPLSA podría ser de utilidad para garantizar los derechos de las PSH.

\section{OBJETIVO}

Estimar la proporción de pediatras de la CABA que conoce la Ley $N^{\circ} 26378$ y recomienda el uso de un IPLSA. Explorar si el conocimiento de la Ley $\mathrm{N}^{\mathrm{o}}$ 26378 se asocia a recomendar a un IPLSA.

\section{MATERIAL Y MÉTODO}

Estudio descriptivo, transversal, por cuestionario semiestructurado, autoadministrado en línea entre el 4/10/19 y el 31/10/19, que utilizó SurveyMonkey TM ${ }^{11}$ (ver Anexo). Se incluyeron pediatras asociados a la Sociedad Argentina de Pediatría (SAP), que ejercieran su actividad en la CABA. La variable de resultado principal fue conocer la Ley $\mathrm{N}^{\circ} 26378$ (sí o no).

Para el segundo objetivo, explorar si el conocimiento de la ley se asociaba a recomendar a un IPLSA, se utilizó la primera como variable de predicción y la segunda como de resultado. Fueron controlados edad, años de práctica médica, sexo, ámbito laboral, experiencia con PSH (ver Anexo).

El estudio fue aprobado por el Comité de Ética en Investigación del Hospital General de Niños "Pedro de Elizalde" y el Comité de Sujetos Humanos de la Junta de Revisión Institucional de la Universidad de Yale. Además, fue avalado por la Subcomisión de Derechos del Niño y aprobado por la Comisión Directiva de la SAP para utilizar su base de datos de correo electrónico para enviar la encuesta.

Al no existir una encuesta validada, fue necesario su desarrollo. Para la validación, se realizaron entrevistas cognitivas a once médicos de un hospital pediátrico que no participaron posteriormente en la encuesta en línea. ${ }^{12}$

Para explorar la asociación entre el conocimiento de la Ley $\mathrm{N}^{\circ} 26378$ y los potenciales predictores, se utilizó la prueba de $\chi^{2}$. Nivel de significación de $\mathrm{p}<0,05$. El análisis se realizó con SPSS 23.0

TABLA 1. Descripción de la población de pediatras que respondieron la encuesta sobre la lengua de señas argentina

\begin{tabular}{|c|c|c|}
\hline Variable & $\mathbf{N}(381)$ & Valor ${ }^{\mathrm{a}}$ \\
\hline Edad & & $45,2 \pm 12,6$ \\
\hline Años de práctica de pediatría & & $19,3 \pm 13,5$ \\
\hline Sexo (mujer/varón) & & $307 / 74$ \\
\hline \multicolumn{3}{|l|}{ Lugar de trabajo } \\
\hline Privado & 103 & $27,1(22,9-31,8)$ \\
\hline Público & 254 & $66,6(61,7-71,1)$ \\
\hline Obras sociales & 24 & $6,3(4,3-9,2)$ \\
\hline Conocen la Ley 26378 & $50 / 331$ & $15,1(11,7-19,4)$ \\
\hline Han atendido a $\mathrm{PSH}^{\mathrm{b}}$ & $335 / 381$ & $87,9(84,3-90,1)$ \\
\hline Puede hablar la LSA correctamente & $15 / 328$ & $4,6(2,8-7,4)$ \\
\hline \multicolumn{3}{|l|}{ Durante la consulta con $\mathrm{PSH}^{c}$} \\
\hline Cuenta con que lean los labios & $265 / 322$ & $82,3(77,8-86,1)$ \\
\hline Cuenta con lenguaje escrito & $165 / 322$ & $51,2(45,8-56,7)$ \\
\hline Utiliza LSA & $42 / 285$ & $14,7(11,1-19,3)$ \\
\hline Cuenta con un IPLSA & $16 / 322$ & $5,0(3,1-7,9)$ \\
\hline Cuenta con un intérprete no profesional & $149 / 322$ & $46,3(40,9-51,7)$ \\
\hline Recomienda a un IPLSA cuando no hay intérprete & $62 / 314$ & $19,7(15,7-24,5)$ \\
\hline Recomienda a un IPLSA cuando hay intérprete no profesional & $15 / 119$ & $12,6(7,8-19,8)$ \\
\hline No conoce la profesión de IPLSA & $101 / 256$ & $39,5(33,7-45,6)$ \\
\hline Desconoce cómo conseguir a un IPLSA & $146 / 256$ & $57,0(50,9-63,0)$ \\
\hline Tuvo problemas para adquirir a un IPLSA & $24 / 272$ & $8,8(6,1-12,8)$ \\
\hline
\end{tabular}

${ }^{a}$ Valores en media \pm desvío estándar (variables continuas) y \% con IC $95 \%$ (variables categóricas).

${ }^{\mathrm{b}}$ Personas sordas o hipoacúsicas (PSH).

"Se consideran los que respondieron "frecuentemente o siempre".

PSH: personas sordas o hipoacúsicas; LSA: Lengua de Señas Argentina; IPLSA: intérprete profesional de Lengua de Señas Argentina. 


\section{RESULTADOS}

Se contactó a 16140 socios registrados en la SAP en todo el país, y se recibieron 1094 respuestas. De estas, 381 correspondían a pediatras con práctica profesional en la CABA, aunque no todos respondieron todas las preguntas de la encuesta. La información sobre las variables analizadas se muestra en Tabla 1.

Respondieron conocer la Ley $\mathrm{N}^{\circ} 26378,50$ de 331 (el 15,1\%; intervalo de confianza-IC- del $95 \%: 11,7-19,4)$. El hecho de conocer la ley se asoció con recomendar a un IPLSA (odds ratio -OR-: 3,7; IC $95 \%$ : 1,1-12,9; p < 0,05), con reportar problemas para conseguirlo (OR: 7,3; IC $95 \%$ : 2,9-18,3; $<<0,01)$ y con estar más familiarizados con la LSA (OR: 5,5; IC $95 \%$ : 2,7-11,0; p <0,001) (Tabla 2).

El 87,9\% de los encuestados respondió haber tenido, al menos, un paciente o padre sordo/ hipoacúsico. En el 92,5\% de los casos en que había un intérprete durante la consulta, se trataba de un intérprete no profesional. Además, 14/25 que intentaron solicitar a un IPLSA tuvieron problemas para conseguirlo (Tabla 1).

\section{DISCUSIÓN}

Nuestro estudio reveló que el 15,1\% de los encuestados (pediatras socios de la SAP que ejercían en la CABA) conocían la Ley $\mathrm{N}^{\circ} 26378$ y que esto estaba asociado a recomendar a un IPLSA. Así como un gran porcentaje de médicos desconocía la ley y no recomendaba a un IPLSA, datos locales mostraron que menos de la mitad de las PSH conocían su derecho a tener a un IPLSA en la consulta médica de sus hijos. ${ }^{10}$

Se observó que más del $95 \%$ de los profesionales encuestados no utilizaban LSA. Esto, sumado a la no utilización de un IPLSA, podría derivar en situaciones embarazosas y predisponer a las PSH a responder "sí a todo", lo que afectaría negativamente la comprensión entre ambos y comprometería el resultado de la consulta médica.

Se observó una asociación entre conocer la Ley $\mathrm{N}^{\circ} 26378$ y recomendar a un IPLSA. Sin embargo, cuando se intentó obtener el servicio de un IPLSA, con frecuencia, no fue exitoso $\mathrm{y}$, en consecuencia, se utilizaron intérpretes no profesionales, quienes podrían no haber

TABLA 2. Descripción de las variables analizadas según el conocimiento de la Ley $N^{o} 26378^{a}$

\begin{tabular}{|c|c|c|c|c|}
\hline \multirow[t]{2}{*}{ Características } & \multicolumn{2}{|c|}{ Conocimiento de la Ley 26378} & \multirow[b]{2}{*}{ OR (IC $95 \%)$} & \multirow[b]{2}{*}{$p$} \\
\hline & Sí $(N=50)$ & No $(N=331)$ & & \\
\hline Edad $^{a}$ & $48,6 \pm 12,4$ & $44,4 \pm 12,6$ & & 0,06 \\
\hline Años de práctica ${ }^{a}$ & $23,1 \pm 13,2$ & $19,1 \pm 13,4$ & & 0,06 \\
\hline Sexo (varón / mujer) & $15 / 35$ & $50 / 229$ & $1,9(0,9-3,8)$ & 0,07 \\
\hline \multicolumn{5}{|l|}{ Lugar de trabajo } \\
\hline Privado & $9(18,4 \%)$ & $81(28,8 \%)$ & & \\
\hline Público & $37(75,5 \%)$ & $183(65,1 \%)$ & & \\
\hline Obras sociales & $3(6,1 \%)$ & $17(6,1 \%)$ & & \\
\hline Haber atendido a $\mathrm{PSH}^{\mathrm{b}}$ (sí/no) & $42 / 8$ & $245 / 36$ & $0,7(0,3-1,7)$ & 0,7 \\
\hline Puede hablar LSA correctamente (sí/no) & $8 / 7$ & $42 / 271$ & $7,3(2,5-21,4)$ & $<0,01$ \\
\hline \multicolumn{5}{|l|}{ Durante la consulta con un PSH } \\
\hline Cuenta con que lean los labios $(\mathrm{s} f / \mathrm{r} n)^{c}$ & $(32 / 10)$ & $(201 / 42)$ & $0,7(0,3-1,4)$ & 0,4 \\
\hline Cuenta con lenguaje escrito ( $\mathrm{f} / \mathrm{r} \mathrm{n}$ ) & $23 / 19$ & $130 / 113$ & $1,1(0,5-2,2)$ & 0,9 \\
\hline Utiliza LSA (s f/r n) & $6 / 36$ & $7 / 236$ & $5,6(1,7-17,6)$ & $<0,01$ \\
\hline Cuenta con un IPLSA (s f/r n) & $6 / 36$ & $8 / 235$ & $4,9(1,6-14,9)$ & $<0,01$ \\
\hline Cuenta con un intérprete no profesional ( $\mathrm{s}$ f/r n) & $16 / 26$ & $117 / 126$ & $0,6(0,4-1,3)$ & 0,2 \\
\hline Recomienda a un IPLSA & & & & \\
\hline cuando no hay intérprete (s f/r n) & $20 / 22$ & $35 / 210$ & $5,4(2,7-11,1)$ & $<0,01$ \\
\hline Recomienda a un IPLSA & & & & \\
\hline cuando hay intérprete no profesional (s f/r n) & $5 / 12$ & $9 / 80$ & $3,7(1,1-12,9)$ & 0,04 \\
\hline Conoce la profesión de IPLSA (sí/no) & $23 / 6$ & $127 / 91$ & $3,6(1,2-11,3)$ & 0,04 \\
\hline Conoce cómo conseguir a un IPLSA (sí/no) & $16 / 13$ & $91 / 127$ & $1,7(0,8-3,7)$ & 0,2 \\
\hline Tuvo problemas para conseguir a un IPLSA (sí/no) & $11 / 30$ & $11 / 220$ & $7,3(2,9-18,3)$ & $<0,01$ \\
\hline
\end{tabular}

a Promedio \pm desvío estándar (prueba de t).

${ }^{\mathrm{b}}$ Persona sorda o hipoacúsica (PSH).

c Siempre o frecuentemente/raramente o nunca ( $\mathrm{s} / \mathrm{r} \mathrm{n})$.

PSH: personas sordas o hipoacúsicas; LSA: Lengua de Señas Argentina; IPLSA: intérprete profesional de Lengua de Señas Argentina; OR: odds ratio; IC: intervalo de confianza. 
interpretado eficazmente la información que daba un médico.

No existen estadísticas que confirmen la cantidad de IPLSA certificados en la Argentina. Estudios en España han demostrado que la cantidad de intérpretes disponibles no logra cubrir la demanda. ${ }^{13}$ De un modo similar, en nuestro país, pocas provincias cuentan con espacios de formación de IPLSA. Para resolver esta dificultad, una alternativa podría ser estimular la capacitación y certificación de IPLSA a nivel nacional.

Este estudio se basó en una muestra de socios de la SAP y, por lo tanto, es vulnerable a un sesgo de selección. A los invitados a participar en la encuesta se les informó el propósito de conocer la utilización de los servicios de intérpretes entre las PSH. De este modo, es posible que la encuesta haya sido respondida en mayor proporción por pediatras con experiencia en el trato a personas con discapacidad (como lo demuestra el alto porcentaje de pediatras que atendieron a pacientes sordos o hipoacúsicos), lo que haría que los resultados reflejaran una situación menos grave de lo que es en realidad.

Los 381 pediatras que respondieron la encuesta representaron un estimado del 13,4 \% de los asociados a la SAP que ejercían su trabajo en la CABA. Esto hizo que la tasa de respuesta en este estudio fuera comparable a las de estudios que utilizaron una metodología similar de muestreo a través de la lista de correo electrónico de la SAP. ${ }^{15}$

Nuestro estudio sugirió que una parte de los pediatras que ejercían en la CABA desconocían la Ley $\mathrm{N}^{\circ} 26378$ y no recomendaban habitualmente a un IPLSA. Se considera necesario concientizar a los profesionales sobre los derechos de las personas con discapacidad, ya que son actores sociales fundamentales para llevar a la práctica las normas legales vigentes.

\section{CONCLUSIONES}

Solo el 15,1\% de los pediatras de la CABA socios de la SAP conoce la Ley $N^{\circ} 26378$. Conocerla se asoció significativamente con recomendar a un IPLSA en la consulta.

\section{REFERENCIAS}

1. GBD 2015 Disease and Injury Incidence and Prevalence Collaborators. Global, regional, and national incidence, prevalence, and years lived with disability for 310 diseases and injuries, 1990-2015: a systematic analysis for the Global Burden of Disease Study 2015. Lancet. 2016;388(10053):1545602.

2. World Health Organization. Deafness and Hearing Loss. Washington DC, 2020. [Acceso: 15 de febrero de 2018]. Disponible en: https: / / www.who.int/news-room/factsheets / detail/ deafness-and-hearing-loss.

3. Argentina. Ministerio de Salud. Audífonos e implantes cocleares gratuitos para promover la integración social de los niños sordos. 2012 [Acceso: 24 de enero de 2018]. Disponible en: http://www.msal.gob.ar/ prensa $/$ index.php?option $=$ com_content\&view $=$ articl e\&id=439:audifonos-e-implantes-cocleares-gratuitospara-promover-la-integracion-social-de-los-ninossordos\&catid=1:noticias439.

4. Emond A, Ridd M, Sutherland H, Allsop L, et al. The current health of the signing Deaf community in the UK compared with the general population: a cross-sectional study. BMJ Open. 2015; 5(1):e006668.

5. Woodcock K, Pole JD. Health profile of deaf Canadians: analysis of the Canada Community Health Survey. Can Fam Physician. 2007; 53(12):2140-1.

6. Barnett S, McKee M, Smith SR, Pearson TA. Deaf sign language users, health inequities, and public health: opportunity for social justice. Prev Chronic Dis. 2011; 8(2):A45.

7. Bat-Chava Y, Martin D, Kosciw JG. Barriers to HIV / AIDS knowledge and prevention among deaf and hard of hearing people. AIDS Care. 2005; 17(5):623-34.

8. Ley N. ${ }^{\circ}$ 27044. Jerarquía Constitucional. Convención sobre los derechos de las personas con discapacidad. Boletín Oficial de la República Argentina. Buenos Aires, 22 de diciembre de2014. [Acceso: 15 deenero de 2018]. Disponible en: http: / / servicios.infoleg.gob.ar / infolegInternet / verNorma.do?id=239860.

9. Convención sobre los Derechos del Niño. Buenos Aires, 27 de setiembre de 1990. [Acceso: 25 de febrero de 2018]. Disponible en: https://www.oas.org/dil/esp/ Convencion_sobre_los_Derechos_del_Nino_Argentina. pdf.

10. Cutri AM, Torres FA, Riquelme CC, Cabrera NB, et al. Prevalencia de personas sordas que cuentan con un intérprete profesional de Lengua de Señas Argentina en la consulta médica de sus hijos. Arch Argent Pediatr. 2018; 116(5):310-5.

11. SurveyMonkey LLC. SurveyMonkey®. 2012. [Acceso: 4 de octubre de 2019]. Disponible en: https: / / es.surveymonkey. com.

12. Willis GB. Cognitive Interviewing: A Tool for Improving Questionnaire Design. California: SAGE Publications, Inc; 2004.

13. Muñoz-Baell IM, Ruiz-Cantero MT, Alvarez-Dardet C, Ferreiro-Lago E, et al. Comunidades sordas: ¿pacientes o ciudadanas? Gac Sanit. 2011; 25(1):72-8.

14. Asch DA, Jedrziewski MK, Christakis NA. Response rates to mail surveys published in medical journals. JClin Epidemiol. 1997; 50(10):1129-36.

15. Goldfarb G, Nasanovsky J, Krynski L, Ciancaglini A, et al. Uso de tecnologías de la información y comunicación por pediatras. Arch Argent Pediatr. 2019; 117(6):S264-76. 


\section{ANEXO \\ Encuesta sobre Lengua de Señas Argentina}

Estimado/a Dr./a.:

La presente encuesta busca evaluar en qué medida los pediatras en nuestro país están al tanto de los derechos de las personas sordas a tener a un intérprete profesional de Lengua de Señas Argentina en las consultas médicas. Esta información resultará de gran valor para la promoción y el diseño de acciones destinadas a una mejor comunicación médico-paciente.

La respuesta a este cuestionario le llevará menos de 5 minutos. Se preservará la confidencialidad de sus respuestas y no guardaremos ningún dato que permita identificarlo.

Si usted está de acuerdo, por favor, haga clic en ACEPTAR para dar su consentimiento y comenzar la encuesta.

Muchas gracias por su participación.

Subcomisión de los Derechos del Niño, Sociedad Argentina de Pediatría

1. ¿Usted es médico pediatra? Sí/No.

2. ¿Dónde ejerce su actividad como médico pediatra? [Puede seleccionar más de una opción].

Ciudad Autónoma de Buenos Aires.

Otro lugar:

3. ¿Cuál es su edad?

4. ¿Hace cuántos años se desempeña como pediatra (incluyendo la residencia/ concurrencia)?

5. ¿Cuál es su género? Varón/Mujer/Otro/No quiere especificar.

6. ¿En qué ámbito desarrolla su mayor carga horaria de trabajo? Institución pública/Institución privada/Obra social.

7. ¿Ha tenido alguna vez pacientes o padres de pacientes sordos o hipoacúsicos? [Puede seleccionar más de una opción].

Sí, pacientes/Sí, padres/No, ni pacientes ni padres.

8. ¿Conoce la Lengua de Señas Argentina (LSA)? Sí/ No.

9. ¿Ha intentado alguna vez aprender Lengua de Señas Argentina (LSA)? Sí/No.

10. ¿Qué tan bien domina la Lengua de Señas Argentina (LSA)?

Muy bien/Bien/No muy bien/Para nada.

11. ¿Tiene algún familiar o amigo sordo o hipoacúsico? Sí/No.

12. Aproximadamente, ¿cuántos pacientes sordos o hipoacúsicos ha tenido?

13. Aproximadamente, ¿cuántos pacientes con padres sordos o hipoacúsicos ha tenido?

14. ¿De qué manera se comunica habitualmente con pacientes sordos o hipoacúsicos o con padres sordos o hipoacúsicos? [Puede seleccionar más de una opción].

Les hablo y ellos leen los labios/Yo uso la Lengua de Señas Argentina/A través de un intérprete profesional de Lengua de Señas Argentina (IPLSA)/ A través de un intérprete no profesional (hijo, familiar, amigo, vecino, etc.)/En forma escrita.

15. Considerando su nivel de dominio de la Lengua de Señas Argentina (LSA), ¿cree que la comunicación entre usted y una persona sorda o hipoacúsica mejoraría con la presencia de un intérprete profesional de Lengua de Señas Argentina (IPLSA)? Sí/ No. 
16. ¿Con qué frecuencia usted cuenta con alguien que actúa de intérprete (profesional o no profesional) entre usted y un paciente sordo o hipoacúsico o con padres sordos o hipoacúsicos? Siempre/Frecuentemente/Raramente/Nunca.

17. En los casos en que hay alguien interpretando, ¿con qué frecuencia esta persona que ocupa el papel de intérprete es un intérprete profesional de Lengua de Señas Argentina (IPLSA)? Siempre/Frecuentemente/Raramente/Nunca.

18. En los casos en que hay alguien interpretando, ¿con qué frecuencia esta persona que ocupa el papel de intérprete es un intérprete no profesional (hijo, familiar, amigo, vecino, etc.)?

Siempre/Frecuentemente/Raramente/Nunca.

19. ¿Con qué frecuencia un paciente sordo o hipoacúsico o con padres sordos o hipoacúsicos traen a un intérprete profesional (IPLSA) a la consulta por sus propios medios?

Siempre/Frecuentemente/ Raramente/Nunca.

20. ¿Con qué frecuencia usted recomienda el uso de un intérprete profesional (IPLSA) con un paciente sordo o hipoacúsico o con padres sordos o hipoacúsicos cuando no hay un intérprete presente? Siempre/Frecuentemente/Raramente/Nunca.

21. ¿Por qué recomendó el uso de un intérprete profesional (IPLSA) en dichos casos? Describa con sus propias palabras:

22. ¿Por qué no recomendó el uso de un intérprete profesional (IPLSA) en dichos casos? [Puede seleccionar más de una opción].

Por desconocimiento de la profesión/ Porque nos podíamos comunicar suficientemente bien sin conseguir a un IPLSA/Por desconocimiento del proceso para conseguir a un IPLSA/Otra (describa con sus propias palabras):

23. ¿Con qué frecuencia recomendó el uso de un intérprete profesional (IPLSA) con un paciente sordo o hipoacúsico o con padres sordos o hipoacúsicos cuando un intérprete no profesional (hijo, familiar, amigo, vecino, etc.) estaba presente?

Siempre/Frecuentemente/Raramente/Nunca.

24. ¿Por qué recomendó el uso de un intérprete profesional (IPLSA) en dichos casos? Describa con sus propias palabras:

25. ¿Por qué no recomendó el uso de un intérprete profesional (IPLSA) en dichos casos? [Puede seleccionar más de una opción].

Por desconocimiento de la profesión.

Porque nos podíamos comunicar suficientemente bien sin conseguir a un IPLSA.

Por desconocimiento del proceso para conseguir a un IPLSA.

Otra (describa con sus propias palabras):

26. ¿Se ha enfrentado alguna vez a algún problema al tratar de conseguir los servicios de un intérprete profesional (IPLSA) después de solicitar uno?

Sí (describa con sus propias palabras):

No.

27. ¿Alguna vez intentó conseguir los servicios de un intérprete no profesional después de tener problemas para solicitar a un intérprete profesional (IPLSA)? Sí/No.

28. ¿Sabe de qué trata la Ley N $\mathrm{N}^{\circ} 26378$ (Convención sobre los Derechos de las Personas con Discapacidad) sobre las personas sordas o hipoacúsicas? Sí/No.

29. ¿Puede describir qué menciona la Ley Nº 26378 sobre las personas sordas e hipoacúsicas?

Describa con sus propias palabras: 